\title{
Increased Risk of Periprosthetic Femur Fractures Associated With a Unique Cementless Stem Design
}

\author{
Chad D. Watts MD, Matthew P. Abdel MD, \\ David G. Lewallen MD, Daniel J. Berry MD, \\ Arlen D. Hanssen MD
}

Received: 21 August 2014 / Accepted: 18 November 2014/Published online: 12 December 2014

(C) The Association of Bone and Joint Surgeons (B) 2014

\begin{abstract}
Background Postoperative periprosthetic femur fractures are an increasing concern after primary total hip arthroplasty (THA). Identifying and understanding predisposing factors are important to mitigating future risk. Femoral stem design may be one such factor.

Questions/purposes The goals of our study were to compare the (1) frequency of periprosthetic femur fracture and implant survivorship; (2) time to fracture in those patients who experienced periprosthetic femur fracture; and (3) predictive risk factors for periprosthetic femur fracture between a unique stem design with an exaggerated proximal taper angle and other contemporary cementless, proximally fixed, tapered stems.

Methods We reviewed all hips in which a femoral hip component with a uniquely exaggerated proximal taper
\end{abstract}

The institution of all authors has received funding from Zimmer (Warsaw, IN, USA), Stryker (Mahwah, NJ, USA), DePuy (Warsaw, IN, USA), and Biomet (Warsaw, IN, USA). One or more of the authors consults for Zimmer (DGL), DePuy (DJB), and Stryker (ADH).

All ICMJE Conflict of Interest Forms for authors and Clinical Orthopaedics and Related Research ${ }^{\circledR}$ editors and board members are on file with the publication and can be viewed on request. Clinical Orthopaedics and Related Research ${ }^{\circledR}$ neither advocates nor endorses the use of any treatment, drug, or device. Readers are encouraged to always seek additional information, including FDA approval status, of any drug or device before clinical use. Each author certifies that his or her institution approved the human protocol for this investigation and that all investigations were conducted in conformity with ethical principles of research.

C. D. Watts, M. P. Abdel, D. G. Lewallen,

D. J. Berry, A. D. Hanssen ( $\square)$

Department of Orthopedic Surgery, Mayo Clinic, 200 First Street

SW, Mayo Clinic, Rochester, MN 55905, USA

e-mail: hanssen.arlen@mayo.edu angle (ProxiLock) was implanted during primary THA at a single academic institution. That group of patients was compared with a cohort of patients who underwent primary THA during the same time interval (1995-2008) in which any other cementless, proximally fixed, tapered stem design was used. The two groups differed somewhat in terms of sex, age, and body mass index, although these differences were of unclear clinical significance. During the study, 3964 primary THAs were performed using six different designs of cementless, proximally fixed, tapered femoral hip prostheses. There were 736 stems in the ProxiLock (PL) patient group and 3228 stems in the nonProxiLock (non-PL) group. In general, the stem highlighted in this study became the routine cementless stem used for primary THA for three arthroplasty surgeons without specific patient or radiographic indications. Periprosthetic fractures were identified within each group. The incidence, timing, type, and treatment required for each fracture were analyzed. The Kaplan-Meier method was used to determine study patient survival free of any postoperative fracture. Radiographs and the electronic medical record of each patient who sustained a fracture were reviewed. Followup was comparable between groups at all time points.

Results The Kaplan-Meier estimate for fracture-free patient survival was worse in the PL group at all time points with survival of $98.4 \%$ (range, 97.4\%-99.3\%), 97.1\% (range, 95.9\%-98.3\%), 95.4\% (range, 93.8\%97.0\%), and $92.6 \%$ (range, $89.6 \%-95.3 \%$ ) at 30 days, 1 year, 5 years, and 10 years, respectively, for the PL patient group compared with $99.8 \%$ (range, 99.7\%99.9\%), 99.6\% (range, 99.3\%-99.8\%), 99.3\% (range, $99.0 \%-99.6 \%$ ), and $98.4 \%$ (range, $97.5 \%-99.1 \%$ ) in the non-PL patient group $(\mathrm{p}<0.001)$. Patients in the PL group had increased cumulative probability of both early and late fractures with cumulative probabilities of fracture of $2.5 \%$ 
(range, $1.3 \%-3.6 \%$ ) at 90 days and $7.4 \%$ (range, $4.7 \%-$ $10.4 \%$ ) at 10 years compared with probabilities of $0.3 \%$ (range, $0.1 \%-0.5 \%$ ) at 90 days and $1.6 \%$ (range, $0.8 \%$ $2.5 \%)$ at 10 years in the non-PL group $(\mathrm{p}<0.001)$. Patients in the PL group had an increased risk of postoperative periprosthetic femur fracture (hazard ratio [HR], 5.6; 95\% confidence interval [CI], 3.4-9.1; $\mathrm{p}<0.001$ ); fracture requiring reoperation (HR, 8.4; 95\% CI, 4.4-15.9); $\mathrm{p}<0.001$ ); and fracture requiring stem revision (HR, 9.1; 95\% CI, 4.5-18.5; $\mathrm{p}<0.001)$. Age older than 60 years was also a risk factor for fracture (HR, 3.7; 95\% CI, 2.1-6.4), but sex, body mass index, and preoperative diagnosis were not predictive.

Conclusions Hips implanted with an uncemented femoral stem, which has a uniquely exaggerated proximal taper angle, had an increased risk of both early and late postoperative periprosthetic femur fracture. The majority of patients with a fracture underwent reoperation or stem revision. The unique proximal geometry, lack of axial support from the smooth cylindrical distal stem as well as resorption of the hydroxyapatite coating and poor ongrowth with subsequent subsidence may contribute to increased risk of fracture. Although this particular stem has recently been discontinued by the manufacturer, these findings are important in regard to followup care for patients with this stem implanted as well as for future cementless stem design in general.

Level of Evidence Level III, therapeutic study.

\section{Introduction}

The use of cementless technology in THA initially gained popularity as complications such as aseptic loosening and "cement disease" began to surface with the use of firstgeneration cement techniques $[4,7,18]$. Although biologic fixation had multiple attractive theoretic benefits, many of the initial cementless femoral stem designs experienced problems, including fixation failure, stress shielding, thigh pain, and osteolysis $[1,2,6]$. To address these issues, newer designs of uncemented femoral prostheses were developed. Currently, up to $90 \%$ of primary THAs in the United States are performed using cementless implants, and contemporary cementless stems have shown long-term implant survival rates as high as $95 \%[3,12]$.

Despite the success of cementless components, periprosthetic femur fractures remain problematic with an increasing frequency resulting from several factors. Foremost, excellent results with cementless THA have led to the use of these implants both in younger, more active patients as well as older patients with osteoporosis [13, 14]. Furthermore, as the trend has moved from cemented to cementless femoral fixation, a higher fracture risk has been identified [13, 14]. Given the relative frequency of fracture with cementless stems and the wide variety of stem designs available, it is conceivable that certain stem characteristics could further contribute to the risk of postoperative fracture. However, few studies have associated specific stem designs with risk for periprosthetic femur fracture [17].

The ProxiLock ${ }^{\circledR}$ (Implex/Zimmer, Warsaw, IN, USA) femoral hip prosthesis has a geometry that is different from other uncemented tapered stems (Fig. 1). The most proximal aspect of this titanium stem has a $14^{\circ}$ circumferential flare ( $7^{\circ}$ per side). This region of the stem is chemically macrotextured and then alumina grit-blasted and hydroxyapatite (HA) coated circumferentially. The remainder of the metaphyseal region of the stem has a $7^{\circ}$ circumferential taper ( $3.5^{\circ}$ per side), which is not chemically treated, but is alumina grit-blasted and HA-coated circumferentially. The distal portion of the stem is a smooth cylinder with an alumina grit "satin" blast surface treatment and no HA coating. According to the manufacturer, the distinctive proximal geometry was designed to create a circumferential seal to minimize particle migration and provide a predictably solid endpoint during implantation. The proximal stem also has an exaggerated medial projection, which is meant to resist medial migration and rotation. The polished distal stem was designed to improve axial load transfer to the proximal

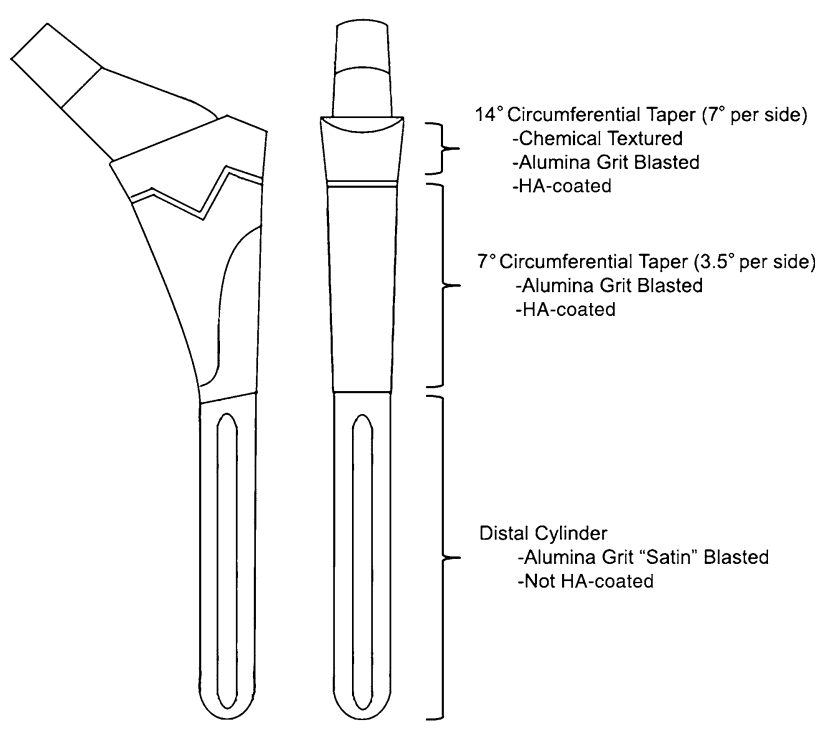

Fig. 1 The ProxiLock stem has a unique geometry from other proximally fixed tapered stems. The most proximal aspect of this titanium stem has a $14^{\circ}$ circumferential flare $\left(7^{\circ}\right.$ per side), which is chemically macrotextured, alumina grit-blasted, and HA-coated circumferentially. The remainder of the metaphyseal region of the stem has a $7^{\circ}$ circumferential taper $\left(3.5^{\circ}\right.$ per side), which is not chemically treated but is alumina grit-blasted and HA-coated circumferentially. The distal portion of the stem is a smooth cylinder with an alumina grit "satin" blast surface treatment and no HA coating. 
wedge. An additional variation of the ProxiLock stem used beaded cobalt-chromium for proximal fixation rather than HA, but no stems of this variety were used at our institution. This stem should not be confused with the identically named ProxiLock femoral hip prosthesis produced by STRATEC Medical (Oberdorf, Switzerland).

The purpose of our study was to compare the (1) frequency of periprosthetic femur fracture and implant survivorship; (2) time to fracture in those patients who experienced periprosthetic femur fracture; and (3) predictive risk factors for periprosthetic femur fracture between ProxiLock stems and other contemporary cementless, proximally fixed tapered stems in patients who underwent THA.

\section{Patients and Methods}

We conducted a single-center, retrospective cohort analysis after obtaining approval from our institutional review board. Our institution's total joint registry, which prospectively captures survival data and patient outcomes, was used to identify all 736 ProxiLock stems that were implanted during primary THA at our institution from 1995 to 2008, all of which were HA-coated (PL group). We then identified a nonPL cohort, which consisted of all other proximally fixed, tapered stems implanted at our institution during the same time interval $(\mathrm{n}=3228)$. Stems were implanted by eight surgeons in the PL group and 25 surgeons in the control group. Three surgeons accounted for 724 (98.4\%) of the stems implanted in the PL group, which encompassed all patients who sustained a postoperative periprosthetic femur fracture. All three were established and dedicated arthroplasty surgeons; two routinely used a direct lateral approach and the other used a posterior approach. Each implanted the ProxiLock as their standard cementless stem for a period of time without specific patient or radiographic indications. However, these same surgeons implanted a large number of non-PL stems and cemented stems during the study period as well. During the study period, Surgeon 1 implanted 174 (19.8\%) PL stems, 309 (35.1\%) non-PL stems, and $398(45.2 \%)$ cemented stems; Surgeon 2 implanted 445 (30.6\%) PL stems, 502 (34.6\%) non-PL stems, and $505(34.8 \%)$ cemented stems; and Surgeon 3 implanted 105 (20.7\%) PL stems, 337 (66.5\%) non-PL stems, and $65(12.8 \%)$ cemented stems. None of these surgeons were designers of the ProxiLock stem.

The two groups differed in age, sex, and body mass index (BMI), although these differences were of unclear clinical significance. There was no difference in indication for primary THA (Table 1). Mean clinical and radiographic followup was 5.8 years (range, 8 days to 15.6 years) for
Table 1. Comparison of study patient characteristics between cohorts

\begin{tabular}{|c|c|c|c|}
\hline Variable & PL group & Non-PL group & $\mathrm{p}$ value \\
\hline Number of patients & 736 & 3228 & 0.002 \\
\hline Number of men $(\%)$ & $448(60.8)$ & $1765(54.7)$ & $<0.001$ \\
\hline Age (years \pm SD) & $60.1 \pm 13$ & $56.7 \pm 13$ & $<0.001$ \\
\hline BMI $\left(\mathrm{kg} / \mathrm{m}^{2} \pm \mathrm{SD}\right)$ & $30.9 \pm 7$ & $30.0 \pm 6$ & 0.48 \\
\hline \multicolumn{4}{|c|}{ Primary diagnosis, number $(\%)$} \\
\hline Osteoarthritis & $551(74.9)$ & $2490(77.1)$ & \\
\hline Avascular necrosis & $97(13.2)$ & $395(12.2)$ & \\
\hline Femoral neck fracture & $38(5.2)$ & $135(4.2)$ & \\
\hline Inflammatory arthritis & $21(2.9)$ & $85(2.6)$ & \\
\hline Posttraumatic & $19(2.6)$ & $70(2.2)$ & \\
\hline Other & $10(1.4)$ & $53(1.6)$ & \\
\hline
\end{tabular}

$\mathrm{PL}=$ ProxiLock; $\mathrm{BMI}=$ body mass index.

the PL group and 5.5 years (range, 12 days to 16.9 years) for the non-PL group. Most recent followup was $\geq 30$ days for 720 (97.8\%) PL patients and 3178 (98.4\%) non-PL patients, $\geq 1$ year for $695(94.4 \%)$ PL patients and 3048 (94.4\%) non-PL patients, $\geq 2$ years for $678(92.1 \%)$ PL patients and $2956(91.6 \%)$ non-PL patients, $\geq 5$ years for $508(69.0 \%)$ PL patients and $2189(67.8 \%)$ non-PL patients, and $\geq 10$ years for $135(18.3 \%)$ PL patients and $559(17.3 \%)$ non-PL patients $(\mathrm{p}<0.001)$. Most patients with limited followup are those whose followup from the primary procedure was truncated as a result of early revision. The non-PL group consisted of 3228 stems, including 50 Synergy ${ }^{\circledR}$ (Smith \& Nephew, Memphis, TN, USA), 95 Trabecular Metal TM ${ }^{\circledR}$ (Zimmer, Warsaw, IN, USA), 237 Omnifit $^{\circledR}$ (Osteonics, Allendale, NJ, USA), 1219 SecurFit $^{\circledR}$ (Stryker, Mahwah, NJ, USA), and 1627 Summit ${ }^{\circledR}$ (DePuy, Warsaw, IN, USA) stems (Table 2). All stems included in this study used axial reaming and broaching for femoral preparation.

Patients who had sustained any periprosthetic femur fracture were identified within each group. Electronic medical records, operative reports, and radiographs were reviewed to examine the timing, fracture pattern, and subsequent treatments. Immediate postoperative radiographs were reviewed for all patients who sustained a postoperative fracture to look for any evidence of missed intraoperative fracture. All fractures were described according to the Vancouver classification, which takes into account fracture location, implant stability, and bone quality [8].

Descriptive statistics are reported as number (percentage), and continuous variables are reported as mean $( \pm \mathrm{SD})$. Univariate Cox proportional hazards regression analysis was performed to assess the association of variables with the risk of any postoperative fracture, fracture requiring reoperation, and fracture requiring stem revision with results reported as hazard ratios (HRs) with 95\% 
Table 2. Stem designs*

\begin{tabular}{|c|c|c|c|c|c|}
\hline Stem & $\begin{array}{l}\text { Number } \\
\text { implanted }\end{array}$ & Metallurgy & Geometry & Proximal surface & Distal surface \\
\hline $\begin{array}{l}\text { ProxiLock } \\
\text { (Implex/Zimmer, } \\
\text { Warsaw, IN, USA) }\end{array}$ & 736 & Titanium & $\begin{array}{l}\text { Distal cylinder, } 3.5^{\circ} \text { circumferential } \\
\text { taper, beginning midstem, } 7^{\circ} \\
\text { proximally circumferential flare }\end{array}$ & $\begin{array}{l}\text { Circumferential chemical } \\
\text { macrotexture, grit-blasted, } \\
\text { HA coating }\end{array}$ & Satin-blasted \\
\hline $\begin{array}{l}\text { Omnifit } \\
\text { (Osteonics, Allendale, } \\
\text { NJ, USA) }\end{array}$ & 237 & Titanium & $3^{\circ}$ biplanar taper, full length & $\begin{array}{l}\text { Circumferentially grit-blasted } \\
\text { with HA coating }\end{array}$ & Coarse \\
\hline $\begin{array}{l}\text { Secur-Fit } \\
\text { (Stryker, Mahwah, NJ, } \\
\text { USA) }\end{array}$ & 1219 & Titanium & $3^{\circ}$ biplanar taper, full length & $\begin{array}{l}\text { Circumferentially arc-deposited } \\
\text { titanium with HA coating }\end{array}$ & $\begin{array}{l}\text { Coarse, } \\
\text { polished tip }\end{array}$ \\
\hline $\begin{array}{l}\text { Summit } \\
\text { (DePuy, Warsaw, IN, } \\
\text { USA) }\end{array}$ & 1627 & Titanium & $3^{\circ}$ biplanar taper, full length & $\begin{array}{l}\text { Circumferentially porocoated } \\
\quad \pm \text { HA coating }\end{array}$ & $\begin{array}{l}\text { Grit-blasted, } \\
\text { polished tip }\end{array}$ \\
\hline $\begin{array}{l}\text { Synergy } \\
\text { (Smith \& Nephew, } \\
\text { Memphis, TN, USA) }\end{array}$ & 50 & Titanium & $3^{\circ}$ biplanar taper, full length & $\begin{array}{l}\text { Circumferentially porous coat } \\
\quad \pm \text { HA coating }\end{array}$ & Grit-blasted \\
\hline $\begin{array}{l}\text { Zimmer TM } \\
\text { (Zimmer, Warsaw, IN, } \\
\text { USA) }\end{array}$ & 95 & Titanium & $\begin{array}{l}3^{\circ} \text { biplanar taper, beginning distally, } 7^{\circ} \\
\text { AP wedge proximally }\end{array}$ & Circumferential trabecular metal & Polished \\
\hline
\end{tabular}

* Although additional variations of stem designs exist, only the details pertaining to the stems implanted in our study are described; $\mathrm{HA}=$ hydroxyapatite.

confidence intervals (CIs). A multivariate analysis was also performed to assess the risk of postoperative fracture with patient variables including group, age, sex, BMI, intraoperative fracture, and preoperative diagnosis. Kaplan-Meier estimates were calculated for patient survival, free of postoperative periprosthetic fractures, fractures requiring reoperation, and fractures requiring revision, with results reported as percentage (95\% CI) [10]. The $\alpha$ level was set at 0.05 for statistical significance.

\section{Results}

Fracture Frequency and Implant Survivorship

Patients in the PL group had an increased incidence of postoperative fracture and worse implant survival than patients in the non-PL group. A total of 39 postoperative fractures were identified in the PL group, including eight Vancouver $A_{G}$, four Vancouver $B_{1}$, and 27 Vancouver $B_{2}$ fractures. In contrast, there were 28 fractures in the non-PL group, including 10 Vancouver $A_{G}$, one Vancouver $A_{L}$, five Vancouver $B_{1}$, and 12 Vancouver $B_{2}$ fractures. The Kaplan-Meier estimate for patient survival, free of any postoperative fracture, was $98.4 \%$ (range, $97.4 \%-99.3 \%$ ), 97.1\% (range, 95.9\%-98.3\%), 95.4\% (range, 93.8\%-
97.0\%), and $92.6 \%$ (range, $89.6 \%-95.3 \%$ ) at 30 days, 1 year, 5 years, and 10 years, respectively, for the PL patient group, compared with $99.8 \%$ (range, 99.7\%99.9\%), 99.6\% (range, 99.3\%-99.8\%), 99.3\% (range, 99.0\%-99.6\%), and $98.4 \%$ (range, $97.5 \%-99.1 \%$ ) in the non-PL patient group ( $\mathrm{p}<0.001$; Table 3$)$.

In addition to having a higher incidence of postoperative fracture, hips in the PL group were more likely to present with a fracture pattern that required surgical treatment. Of the 39 fractures in the PL group, 30 (76\%) were treated surgically, including use of open reduction internal fixation (ORIF) in five of $39(13 \%)$ fractures and stem revision in 25 of $39(64.1 \%)$ fractures. In contrast, 14 of $28(50 \%)$ fractures were treated operatively in the non-PL group, including three of $28(11 \%)$ treated with ORIF and 11 of 28 fractures (39\%) treated with stem revision $(\mathrm{p}<0.001)$. Kaplan-Meier estimates for patient survival, free of reoperation and stem revision, are shown (Table 3). At the time of revision for fracture, we routinely found HA resorption and poor ongrowth associated with the ProxiLock stem (Fig. 2).

At latest followup, 33 (4.5\%) stem revisions had been performed in the PL group with diagnoses including fracture, 25 (3.4\%); symptomatic loosening, seven (1\%); and infection, one $(0.1 \%)$. In the non-PL group, fewer stems were revised (32 [1\%]), including $15(0.5 \%)$ for infection, $11(0.3 \%)$ for fracture, and six $(0.2 \%)$ for symptomatic loosening $(\mathrm{p}<0.001)$. 
Table 3. Estimated Kaplan-Meier component survival for both study patient groups

\begin{tabular}{|c|c|c|c|c|c|}
\hline Fracture/cohort & 30 days $(\%)$ & 1 year $(\%)$ & 5 years $(\%)$ & 10 years $(\%)$ & $\mathrm{p}$ value \\
\hline \multicolumn{6}{|c|}{ Any postoperative fracture } \\
\hline PL & $98.4(97.4-99.3)$ & 97.1 (95.9-98.3) & $95.4(93.8-97.0)$ & $92.6(89.6-95.3)$ & \multirow[t]{2}{*}{$<0.001$} \\
\hline Non-PL & $99.8(99.7-99.9)$ & $99.6(99.3-99.8)$ & $99.3(99.0-99.6)$ & $98.4(97.5-99.1)$ & \\
\hline \multicolumn{6}{|c|}{ Fracture requiring reoperation } \\
\hline PL & $98.6(97.8-99.5)$ & $97.8(96.8-98.9)$ & $96.5(95.2-97.9)$ & $94.2(91.6-96.7)$ & \multirow[t]{2}{*}{$<0.001$} \\
\hline Non-PL & $99.8(99.7-99.9)$ & $99.8(99.7-99.9)$ & $99.7(99.5-99.9)$ & 98.9 (98.2-99.6) & \\
\hline \multicolumn{6}{|c|}{ Fracture requiring revision } \\
\hline PL & $98.6(97.8-99.5)$ & $97.7(96.6-98.8)$ & $97.0(95.8-98.3)$ & $95.2(92.7-97.4)$ & \multirow[t]{2}{*}{$<0.001$} \\
\hline Non-PL & $99.8(99.7-99.9)$ & $99.8(99.7-99.9)$ & $99.8(99.6-99.9)$ & $99.2(98.5-99.8)$ & \\
\hline
\end{tabular}

Values per Kaplan-Meier survival estimate (95\% confidence interval); $\mathrm{p}$ values were determined using Cox model analysis; PL $=$ ProxiLock.

Fig. 2A-B (A) This radiograph demonstrates a late Vancouver $\mathrm{B}_{2}$ fracture, which occurred after a ground-level fall in a patient with a ProxiLock stem. (B) When this patient underwent revision, we noted HA resorption and poor ongrowth, which were common findings in the PL group during revision for late Vancouver $B_{2}$ fractures.
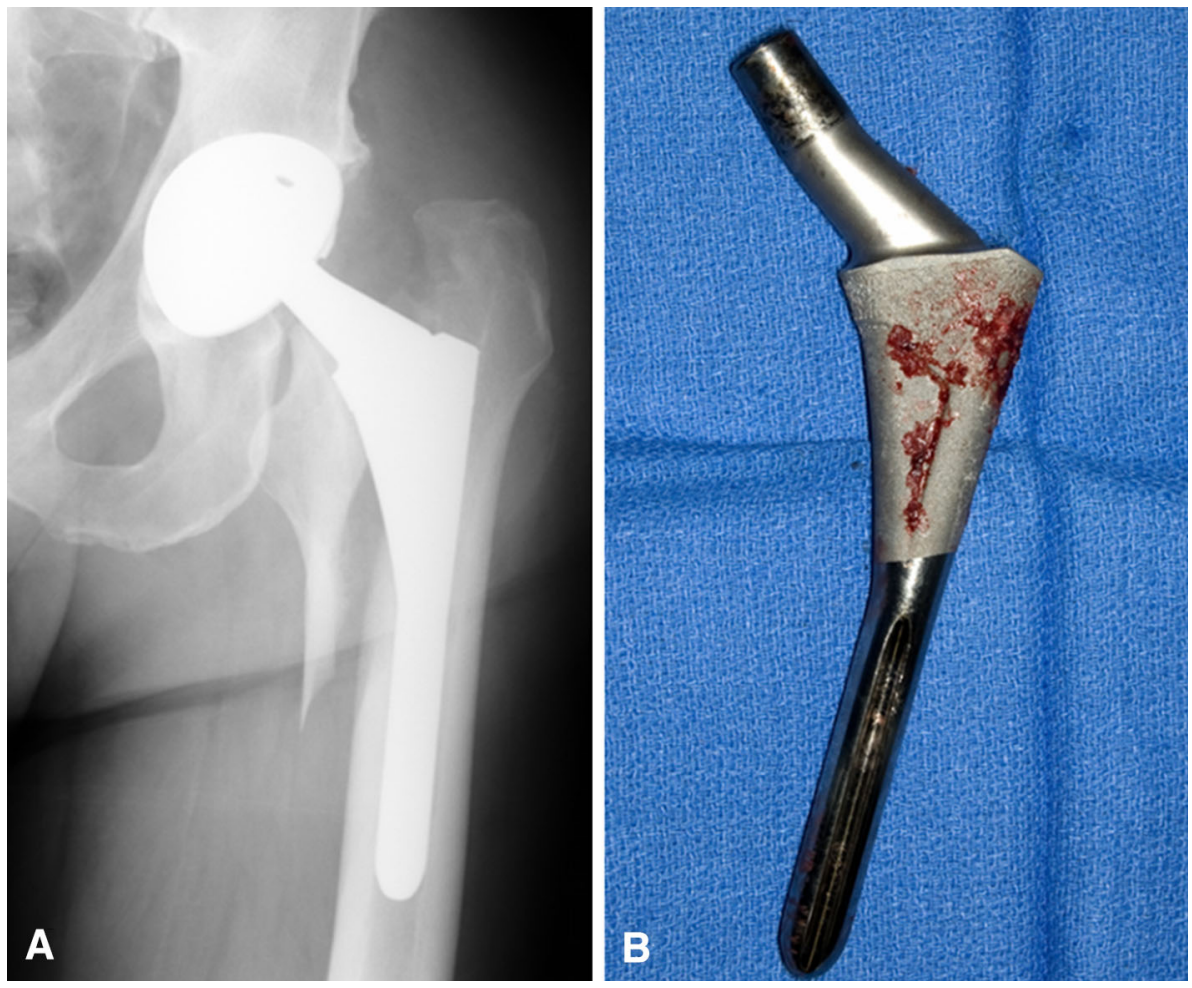

\section{Time to Fracture}

An increased fracture probability was present in the PL group at all time points encompassing both early and late fractures. In the PL group, the cumulative probability of fracture was $1.6 \%(0.7 \%-2.6 \%)$ at 30 days, $2.5 \%(1.3 \%-$ $3.6 \%)$ at 90 days, $2.9 \%(1.7 \%-4.1 \%)$ at 1 year, $4.6 \%$ $(3.0 \%-6.2 \%)$ at 5 years, and $7.4 \%(4.7 \%-10.4 \%)$ at 10 years. In the non-PL group, the cumulative probability of fracture was $0.2 \%(0.04 \%-0.3 \%)$ at 30 days, $0.3 \%$ $(0.1 \%-0.5 \%)$ at 90 days, $0.4 \%(0.2 \%-0.7 \%)$ at 1 year, $0.7 \%(0.04 \%-1 \%)$ at 5 years, and $1.6 \%(0.8 \%-2.5 \%)$ at 10 years $(\mathrm{p}<0.001$; Fig 3$)$.
Patient characteristics were similar between patients who sustained early ( $\leq 90$ days) and late ( $>90$ days) fractures within the PL group with regard to age $(64.6 \pm 12.7$ years versus $68.5 \pm 11.4$ years, $p=0.32)$ and female sex $(61.1 \%$ versus $52.4 \%, \mathrm{p}=0.41)$. Furthermore, fracture type was also similar between early and late fractures within the PL group. Of 18 early fractures, four (22.2\%) were Vancouver $\mathrm{A}_{\mathrm{G}}$ and 14 (77.8\%) were Vancouver $\mathrm{B}_{2}$, similar to the 21 late fractures, which consisted of four (19\%) Vancouver $A_{G}$, four (19\%) Vancouver $B_{1}$, and $13(62 \%)$ Vancouver $B_{2}(p=0.82)$. Within the nonPL group, age was similar between patients with early and late fractures $(67.4 \pm 12.9$ years vs $62.7 \pm 10.2$ years, 


\section{Cumulative Probability of Postoperative Fracture}

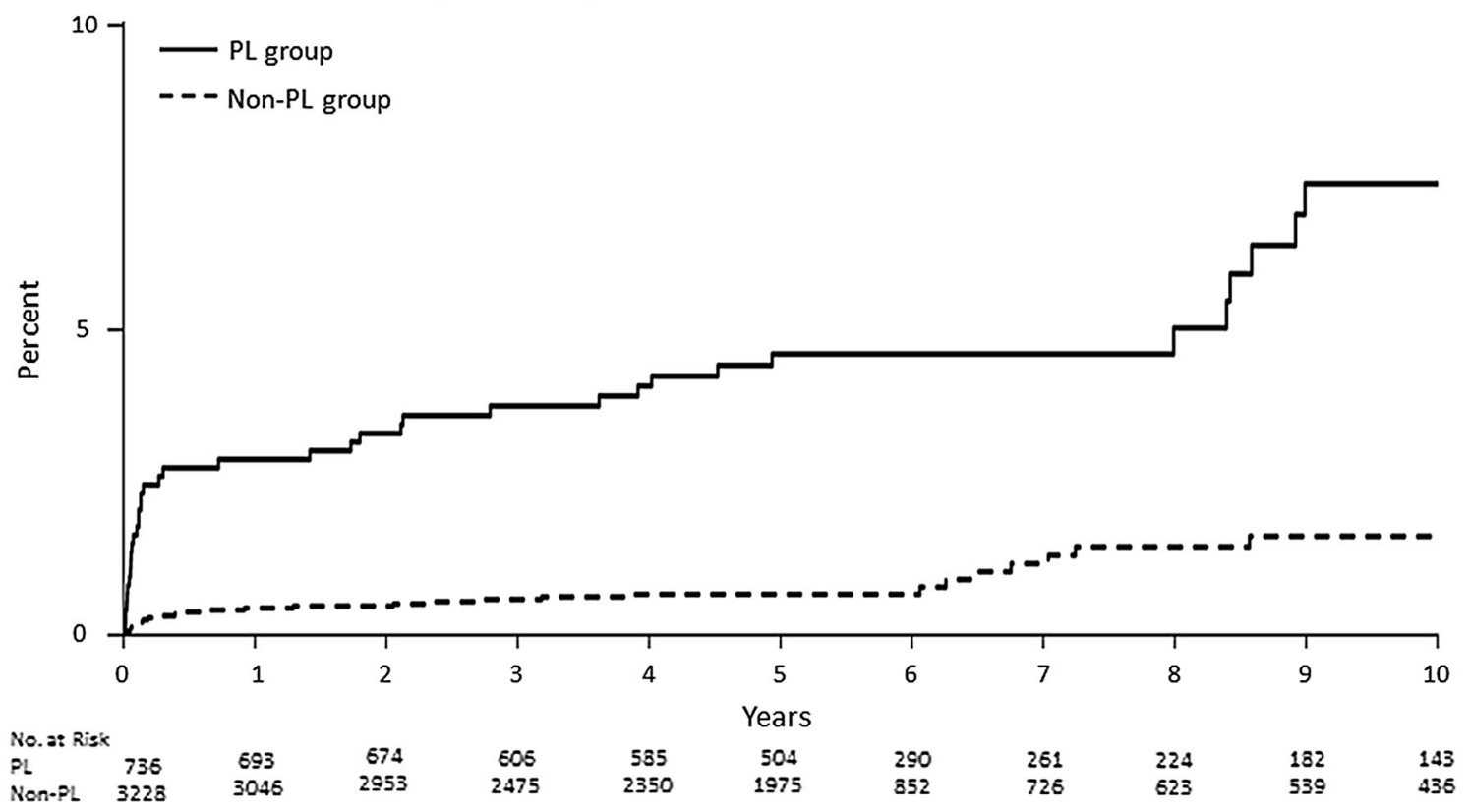

Fig. 3 Cumulative probability estimates demonstrate that there was an increased probability of postoperative fracture at all time points in the PL group (solid line) when compared with the non-PL group (dashed line).

$\mathrm{p}=0.30$ ), but there tended to be a higher proportion of females among the early fractures than late fractures $(66.7 \%$ versus $31.6 \%, p=0.08)$. Of nine early fractures in the non-PL group, two $(22.2 \%)$ were Vancouver $\mathrm{A}_{\mathrm{G}}$, one $(11.1 \%)$ was a Vancouver $B_{1}$, and six $(66.7 \%)$ were Vancouver $\mathrm{B}_{2}$. In contrast, 19 late fractures consisted of eight (42.1\%) Vancouver $A_{G}$, one (5.3\%) Vancouver $A_{L}$, four $(21.1 \%)$ Vancouver $\mathrm{B}_{1}$, and six $(31.6 \%)$ Vancouver $\mathrm{B}_{2}$ $(\mathrm{p}=0.12)$.

\section{Predictive Risk Factors}

Use of a ProxiLock stem and age older than 60 years were risk factors for postoperative fracture, fracture requiring reoperation, and fracture requiring stem revision. After controlling for confounding variables including patient sex, age, BMI, primary diagnosis, and intraoperative fracture, use of a ProxiLock stem demonstrated an increased risk of postoperative fracture (HR, 5.0; 95\% CI, 3.1- 8.2; $\mathrm{p}<0.001)$. Age older than 60 years was also a risk factor (HR, 3.7; 95\% CI, 2.1-6.4; p < 0.001). Patient sex, BMI, and preoperative diagnosis were not risk factors (Table 4).

There were $36(4.9 \%)$ recognized intraoperative fractures in the PL group, which was similar to the rate in the non-PL group (139 [4.3\%]). Use of a ProxiLock stem did not lead to increased risk of intraoperative fracture (HR, $1.1 ; 95 \% \mathrm{CI}, 0.8-1.7 ; \mathrm{p}=0.48)$. Of the $36 \mathrm{PL}$ group patients who sustained an intraoperative fracture, three developed a subsequent postoperative fracture. In one instance, a nondisplaced calcar crack was treated with a single Luque wire. The patient did well until sustaining a Vancouver $B_{1}$ fracture after a ground-level fall 4 years postoperatively, which was treated with ORIF. In another instance, a small trochanteric avulsion fracture occurred during hip reduction and was treated with suture repair and a postoperative hip abduction brace, but the patient fell and sustained a Vancouver $\mathrm{B}_{2}$ fracture requiring stem revision 8 weeks postoperatively. In the third case, a trochanteric crack was noted during preparation of the femoral canal, which was repaired using Dacron tape suture, but the patient was involved in a tractor rollover accident 3 weeks postoperatively, which resulted in a Vancouver $B_{2}$ fracture, requiring stem revision. After reviewing the immediate postoperative radiographs for all patients who sustained a postoperative fracture, we were unable to identify any unrecognized intraoperative fractures.

\section{Discussion}

Cementless, proximally fixed, tapered femoral components have been among the most frequently used for primary THA. Their design relies on press-fit and three-point fixation to achieve initial stability. Because the viscoelastic proximal femur then gradually accommodates to the pressfit, implant subsidence is prevented by three-point fixation, the tapered geometry, and surface treatments [16]. Stability 
Table 4. Analysis of risk factors for any postoperative fracture

\begin{tabular}{|c|c|c|c|c|}
\hline \multirow[t]{2}{*}{ Factor } & \multicolumn{2}{|c|}{ Univariate analysis } & \multicolumn{2}{|c|}{ Multivariate analysis } \\
\hline & HR $(95 \% \mathrm{CI})$ & $\mathrm{p}$ value & HR $(95 \%$ CI $)$ & $\mathrm{p}$ value \\
\hline \multicolumn{5}{|l|}{ Group } \\
\hline PL & $5.6(3.4-9.1)$ & $<0.001$ & $5.0(3.1-8.2)$ & $<0.001$ \\
\hline Non-PL & 1.0 (reference) & & 1.0 (reference) & \\
\hline \multicolumn{5}{|l|}{ Age (years) } \\
\hline$>60$ & $3.7(2.1-6.4)$ & $<0.001$ & $3.6(2.0-6.3)$ & $<0.001$ \\
\hline$\leq 60$ & 1.0 (reference) & & 1.0 (reference) & \\
\hline \multicolumn{5}{|l|}{ Sex } \\
\hline Women & $1.3(0.8-2.2)$ & 0.23 & $1.5(0.96-2.5)$ & 0.08 \\
\hline Men & 1.0 (reference) & & 1.0 (reference) & \\
\hline \multicolumn{5}{|l|}{ BMI $\left(\mathrm{kg} / \mathrm{m}^{2}\right)$} \\
\hline$\geq 30$ & $1.0(0.6-1.6)$ & 0.98 & $1.0(0.6-1.6)$ & 0.98 \\
\hline$<30$ & 1.0 (reference) & & 1.0 (reference) & \\
\hline \multicolumn{5}{|l|}{ Intraoperative fracture } \\
\hline Yes & $1.2(0.4-3.6)$ & 0.75 & $1.3(0.5-3.5)$ & 0.65 \\
\hline No & 1.0 (reference) & & 1.0 (reference) & \\
\hline \multicolumn{5}{|l|}{ Diagnosis } \\
\hline AVN & $1.5(0.8-2.8)$ & 0.79 & $2.1(1.1-4.1)$ & 0.27 \\
\hline Femoral neck fracture & $1.5(0.5-4.0)$ & & $1.8(0.6-5.0)$ & \\
\hline Inflammatory arthritis & $0.6(0.1-4.3)$ & & $0.8(0.1-6.2)$ & \\
\hline Posttraumatic arthritis & $1.4(0.4-5.9)$ & & $1.7(0.2-12.4)$ & \\
\hline Other & $1.1(0.2-8.1)$ & & $1.9(0.5-7.8)$ & \\
\hline Osteoarthritis & 1.0 (reference) & & 1.0 (reference) & \\
\hline
\end{tabular}

$\mathrm{HR}=$ hazard ratio; $\mathrm{CI}=$ confidence interval; $\mathrm{PL}=$ ProxiLock; $\mathrm{BMI}=$ body mass index; $\mathrm{AVN}=$ avascular necrosis.

must be maintained in this manner with micromotion less than $20 \mu \mathrm{m}$ until biologic fixation is obtained, a process that may require up to 12 weeks [9]. Gradual loading of the proximal femur and tapered geometries have resulted in reduced stress shielding and thigh pain, respectively [12, 16]. Despite advances made in THA surgical technique and implant technology, periprosthetic fracture continues to be a problem causing severe patient morbidity [5, 13-15]. Although several studies have previously reported on the epidemiology of periprosthetic femur fracture, there are little data pertaining to the fracture risk of particular cementless stems. Sheth et al found that metaphyseal engaging stems, especially flat tapered stems, led to an increased risk of periprosthetic femur fracture compared with cemented and diaphyseal engaging stems [17]. However, we are not aware of any studies that describe variations of fracture risk within the same class of cementless femoral stems. The aims of our study were to determine the (1) frequency of periprosthetic femur fracture and implant survivorship; (2) time to fracture in those patients who experienced periprosthetic femur fracture; and (3) predictive risk factors for periprosthetic femur fracture between ProxiLock stems and other contemporary cementless, proximally fixed tapered stems in patients who underwent primary THA.

There are limitations to our study. Foremost, although our data were prospectively collected, the data were examined retrospectively. Because of this, it is possible that important differences existed between the PL and nonPL groups. For example, patients in the PL group were found to be older with a higher proportion of males. Although these differences were small and not likely to be clinically important, it is possible there are other differences that were not identified by this study such as differences in femoral bony geometry or nuanced surgeon indications for use of the ProxiLock stem, which may have influenced the risk of fracture. Furthermore, our study cohorts were not matched; however, to make our comparisons as applicable as possible, the non-PL group included only uncemented stems that were tapered, proximally fixed, and implanted during the same period of time as the PL group. Additionally, our ability to definitively explain the cause of fracture, especially late fracture, is limited because radiographs were not routinely taken within a short time preceding fracture, which makes it impossible to describe findings such as loosening, 
subsidence, or osteolysis, which could predispose to fracture. Lastly, because no cobalt-chromium beaded versions of the PL stem were implanted at our institution, our results only apply to the HA-coated version.

The prevalence of postoperative periprosthetic femur fracture after primary THA has previously been described as $0.4 \%$ to $1.1 \%$ [5, 11, 13, 14]. Although the prevalence of fracture in our non-PL group patients was comparable to this rate $(0.87 \%)$, in our study, fractures were much more common in the PL group (5.3\%). More importantly, a larger proportion of PL patients presented with fracture types that required reoperation or stem revision. Fracture was the second most common reason for revision in the non-PL group, accounting for 11 of 32 (34.4\%) of revisions. However, in the PL group, periprosthetic fracture was the number one reason for stem revision, accounting for 25 of $33(75.8 \%)$ revisions.

We found that an increased probability of fracture was present at all time points in the PL group compared with other cementless stems, including both early and late fractures. The timing of postoperative fractures has important implications. Early fractures are most likely the result of missed intraoperative fractures or stem subsidence before osseointegration. Because the rate of intraoperative fracture was similar between the PL and non-PL groups and no cases of missed intraoperative fracture could be identified after reviewing immediate postoperative radiographs, it would seem unlikely that missed intraoperative fractures account for the increased risk of early postoperative fractures in the PL group. In regard to late fractures, it is somewhat puzzling why an increased risk would exist once a cementless stem has theoretically attained biologic osseointegration. However, epidemiologic studies demonstrate that cementless stems are in fact associated with an increased risk of late fracture compared to cemented stems $[14,15,17]$. With this in mind, late fractures could be the result of diminishing bone quality, osteolysis, or stem loosening/failure to osseointegrate and subsidence.

Our data showed that use of the HA-coated ProxiLock stem was a risk factor for postoperative periprosthetic femur fracture (HR, 5.6), fracture requiring reoperation (HR, 8.4), and fracture requiring stem revision (HR, 9.1) compared with all other cementless, proximally fixed tapered stems implanted at our institution during the same time interval. When taking all stem designs into consideration, patient age older than 60 years was also a risk factor for the same outcomes with HRs of 3.7, 4.2, and 4.5, respectively. Our findings are consistent with a study by Cook et al, which noted that patients aged older than 70 years had a 2.9 times greater risk of periprosthetic femur fracture, but found no increased risk associated with female sex (HR 1.5 in our study) [5]. Intraoperative fractures occurred with similar frequency in both of our study groups and did not increase the risk of postoperative fractures.

Although we are not able to definitively explain the reason for the increased frequency of fracture in the ProxiLock group, we propose that multiple factors pertaining to the stem itself may contribute. Foremost, the stem geometry is unique. Although the majority of threedimensional tapered stems use a $3^{\circ}$ taper that spans the entire stem length, the ProxiLock taper has two distinct transition points: from a smooth distal cylinder to a $3.5^{\circ}$ per side circumferential taper at midstem and then to a $7^{\circ}$ per side circumferential flare proximally. For early fractures (before osseointegration), this pronounced double wedge could predispose to fracture in the setting of an axial force or subsidence, especially with a polished distal cylinder, which provides less axial support than stems that are gritblasted and tapered distally. Explaining late fractures is made difficult by the lack of radiographs immediately preceding fracture. However, we found that most late fractures in the PL group were Vancouver $B_{2}$ fractures with HA resorption and poor ongrowth but little or no osteolysis noted at the time of revision. This leads us to suggest that HA resorption compounded by lack of distal fixation and an exaggerated proximal taper may lead to gradual loosening and subsidence with a subsequent increased risk of late periprosthetic femur fracture.

In summary, use of the HA-coated ProxiLock stem in THA is associated with a high rate of postoperative periprosthetic femur fracture and, importantly, with fracture types necessitating reoperation or stem revision. The ProxiLock stem has not been used at our institution since 2008 , and it has recently been discontinued by the manufacturer. Nevertheless, the findings reported in this study are important considerations for patients with existing ProxiLock stems and may be especially valid for those with evidence of loosening or stem subsidence. Furthermore, these findings are important in regard to future implant design. Specific design features that may increase the risk of fracture include exaggerated proximal tapers, lack of axial support from the distal stem, and HA coating on relatively smooth ongrowth surfaces. Additional understanding could be gleaned from retrieval analysis of failed HA-coated ProxiLock stems or outcome studies of the beaded cobalt-chrome version.

Acknowledgments We thank Youlonda A. Loechler for her assistance in the data collection and W. Scott Harmsen and Kristin Fruth for their statistical expertise.

\section{References}

1. Callaghan JJ, Dysart SH, Savory CG. The uncemented porouscoated anatomic total hip prosthesis. Two-year results of a 
prospective consecutive series. J Bone Joint Surg Am. 1988; 70:337-346.

2. Campbell D, Mercer G, Nilsson KG, Wells V, Field JR, Callary SA. Early migration characteristics of a hydroxyapatite-coated femoral stem: an RSA study. Int Orthop. 2011;35:483-488.

3. Capello WN, D'Antonio JA, Jaffe WL, Geesink RG, Manley MT, Feinberg Jr. Hydroxyapatite-coated femoral components: 15-year minimum followup. Clin Orthop Relat Res. 2006;453:75-80.

4. Collis DK. Cemented total hip replacement in patients who are less than fifty years old. J Bone Joint Surg Am. 1984;66:353-359.

5. Cook RE, Jenkins PJ, Walmsley PJ, Patton JT, Robinson CM. Risk factors for periprosthetic fractures of the hip: a survivorship analysis. Clin Orthop Relat Res. 2008;466:1652-1656.

6. Dodge BM, Fitzrandolph R, Collins DN. Noncemented porouscoated anatomic total hip arthroplasty. Clin Orthop Relat Res. 1991;269:16-24.

7. Dorr LD, Takei GK, Conaty JP. Total hip arthroplasties in patients less than forty-five years old. J Bone Joint Surg Am. 1983;65:474-479,

8. Duncan CP, Masri BA. Fractures of the femur after hip replacement. Instr Course Lect. 1995;44:293-304.

9. Jasty M, Bragdon C, Burke D, O'Connor D, Lowenstein J, Harris WH. In vivo skeletal responses to porous-surfaced implants subjected to small induced motions. J Bone Joint Surg Am. 1997;79:707-714.

10. Kaplan EL, Meier P. Nonparametric estimation from incomplete observations. Am Stat Assoc. 1958;53:457-481.
11. Kavanagh BF. Femoral fractures associated with total hip arthroplasty. Orthop Clin North Am. 1992;23:249-257.

12. Khanuja HS, Vakil JJ, Goddard MS, Mont MA. Cementless femoral fixation in total hip arthroplasty. J Bone Joint Surg Am. 2011;93:500-509.

13. Lewallen DG, Berry DJ. Periprosthetic fracture of the femur after total hip arthroplasty: treatment and results to date. Instr Course Lect. 1998;47:243-249.

14. Lindahl H, Malchau H, Herberts P, Garellick G. Periprosthetic femoral fractures classification and demographics of 1049 periprosthetic femoral fractures from the Swedish National Hip Arthroplasty Register. J Arthroplasty. 2005;20:857-865.

15. Lindahl H, Oden A, Garellick G, Malchau H. The excess mortality due to periprosthetic femur fracture. A study from the Swedish national hip arthroplasty register. Bone. 2007;40: 1294-1298.

16. Mallory TH, Head WC, Lombardi AV Jr. Tapered design for the cementless total hip arthroplasty femoral component. Clin Orthop Relat Res. 1997;344:172-178.

17. Sheth NP, Brown NM, Moric M, Berger RA, Della Valle CJ. Operative treatment of early peri-prosthetic femur fractures following primary total hip arthroplasty. J Arthroplasty. 2013;28: 286-291.

18. Sutherland CJ, Wilde AH, Borden LS, Marks KE. A ten-year follow-up of one hundred consecutive Muller curved-stem total hip-replacement arthroplasties. J Bone Joint Surg Am. 1982;64: 970-982. 